\title{
Atypical Tracheobronchial Vascular Compression
}

\author{
Brian J. Wiatrak, MD, Charles M. Myer III, MD, and Robin T. Cótton, MD
}

\begin{abstract}
Vascular compression of the tracheobronchial tree frequently presents early in infancy with significant airway compromise. For this reason, the pediatric otolaryngologist is often consulted early in the assessment of these patients. Three unusual cases of tracheobronchial vascular compression are presented. The diagnosis and management of children with tracheobronchial vascular compression is discussed, stressing the importance of synchronous airway anomalies and associated congenital cardiac anomalies. Although surgical intervention may be corrective in most cases of vascular compression, persistent tracheomalacia may necessitate tracheotomy for a prolonged period. AM J OTOLARYNGOL 12:347-356. Copyright (C) 1991 by W.B. Saunders Company

Key words: tracheobronchial, vascular ring, cardiac anomalies.
\end{abstract}

It has been estimated that approximately $3 \%$ of the general population have anomalies involving the great vessels or the mediastinum, but only a small portion of these result in symptomatic vascular compression of the airway. ${ }^{1}$ The most symptomatic anomalies are vascular rings that completely encircle the trachea and esophagus. However, vascular slings, which are noncircumferential vascular anomalies, may also lead to significant morbidity and, occasionally, mortality. The most common vascular ring anomaly is a double aortic arch, while the most common vascular sling causing airway compression is innominate artery compression of the trachea. ${ }^{2-6}$

Vascular compression of the airway should be considered in any neonate with evidence of airway obstruction. The initial investigation should include flexible laryngoscopy as well as chest and neck radiographs. A chest radiograph is mandatory to rule out cardiomegaly and to assess the pulmonary artery vasculature. Barium swallow and airway fluoroscopy may be used to identify evidence of vascular compression. If such a lesion is suspected, a complete evaluation of the tracheo-

Received April 13, 1991 from the Department of Otolaryngology-Head and Neck Surgery, University of Michigan, Ann Arbor, MI; and the Department of Otolaryngology and Maxillofacial Surgery, Children's Hospital Medical Center, Cincinnati, OH. Accepted for publication October 9, 1991.

Presented at the International Congress of Pediatric Otolaryngology, Ghent, Belgium, June, 1990.

Address correspondence and reprint requests to Brian J. Wiatrak, MD, 2451 Taubman Center, Box 0313, 1500 E Medical Center Dr, Ann Arbor, MI 48109-0313

Copyright (C) 1991 by W.B. Saunders Company

0196-0709/91/1206-0002\$5.00/0 bronchial tree should be performed endoscopically, looking for the location and degree of obstruction. In addition, other synchronous airway lesions, including vocal cord paralysis and laryngotracheal stenosis, should be actively investigated. In our experience, the combination of rigid endoscopy and magnetic resonance imaging (MRI) is the diagnostic modality of choice. ${ }^{7-9}$ Surgical intervention to relieve vascular compression may be insufficient due to the presence of significant tracheobronchomalacia. In such cases, longterm tracheotomy may be necessary.

We present three atypical cases of tracheobronchial vascular compression necessitating surgical intervention. The embryology of the vascular arch derivatives is discussed, as well as the diagnosis and therapeutic management of vascular compression of the tracheobronchial tree.

\section{CASE REPORTS}

Case report no. 1. A premature infant of 35-week gestational age, born with an omphalocele, was intubated immediately after birth for respiratory distress. The omphalocele was repaired soon after birth; however, due to severe pulmonary insufficiency and the development of bronchopulmonary dysplasia, the patient underwent tracheotomy after a prolonged course of nasotracheal intubation. He was referred to Children's Hospital, Cincinnati, $\mathrm{OH}$ at 5 months of age for further evaluation. Microlaryngoscopy and bronchoscopy demonstrated severe distal tracheomalacia and proximal left mainstem bronchomalacia (Fig 1). Magnetic resonance imaging scan ( $F$ ig 2) revealed an anomalous descending aorta located in the retroesophageal position, compressing the trachea and left mainstem bronchus. 'The aortic arch was noted to be normal, arising on the left side. The patient subsequently underwent an aortopexy through a left thoracotomy to later- 


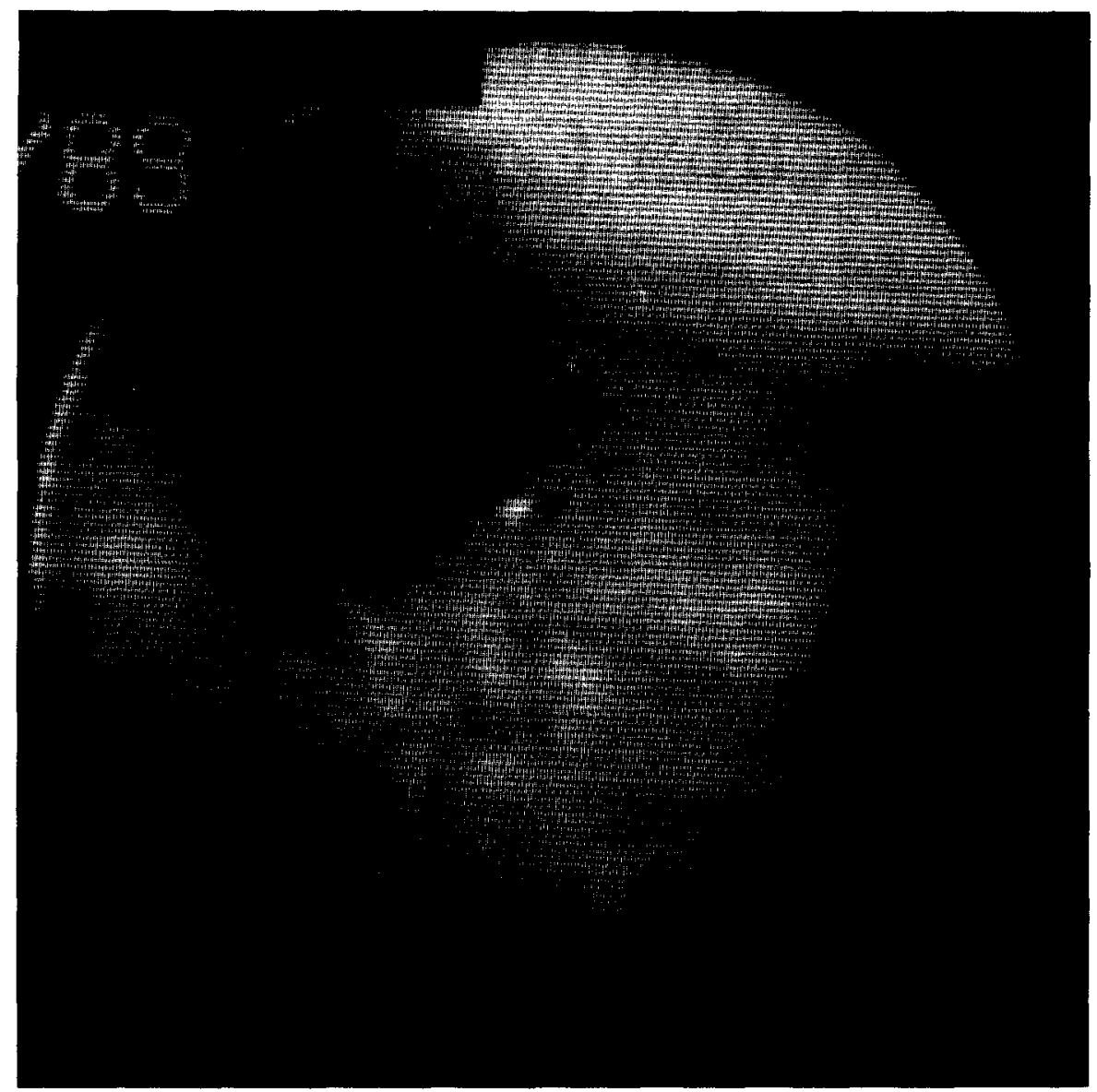

Figure 1. Endoscopic appearance of proximal left mainstem bronchus demonstrating severe bronchomalacia.

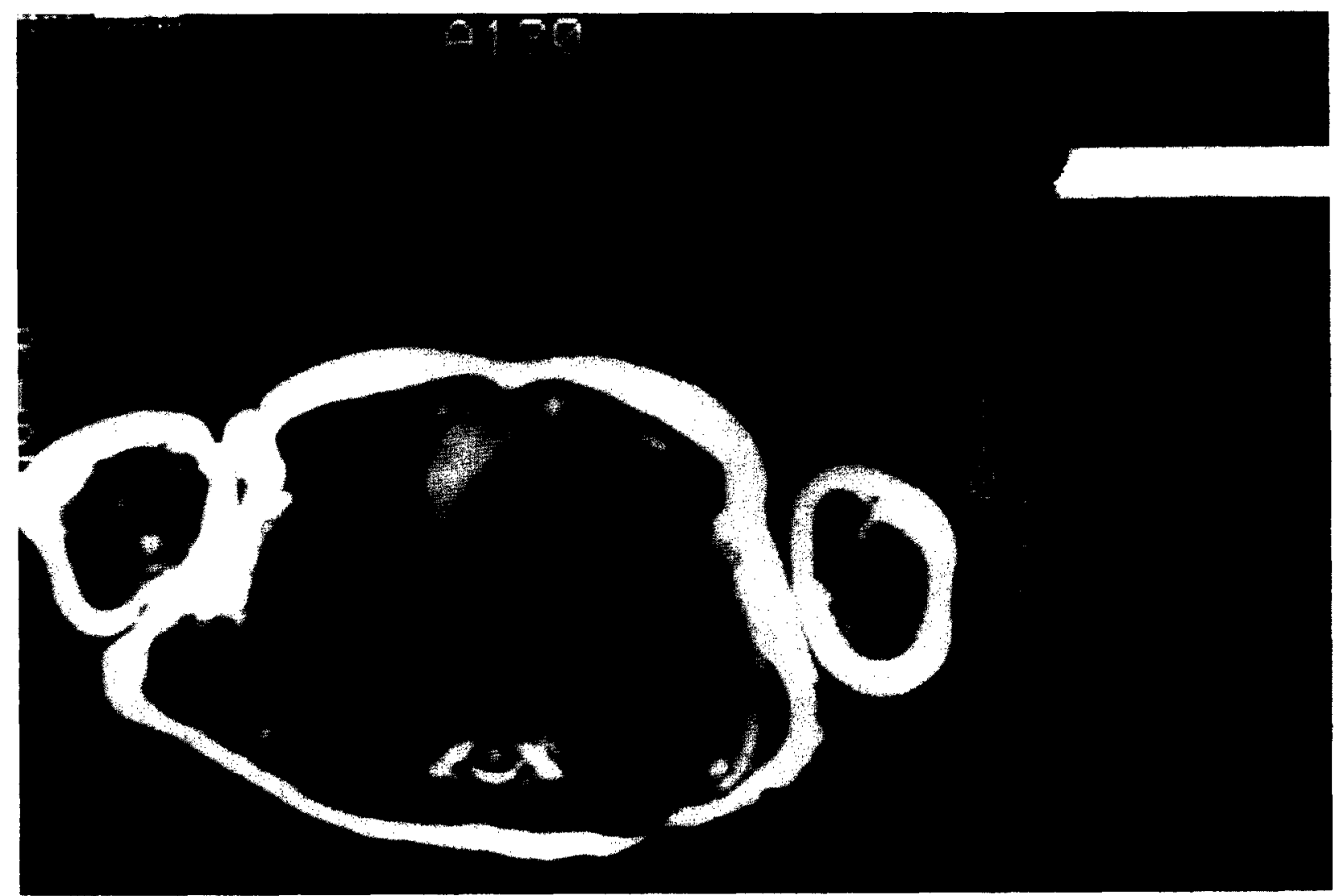

Figure 2. Magnetic resonance imaging scan of an anomalous descending retroesophageal aorta compressing the esophagus and trachea posteriorly. 
alize the aorta, resulting in minimal improvement. The patient continues with a tracheotomy and remains ventilator-dependent 10 months after surgery.

Case report no. 2. A 9-day-old black male of term gestation underwent a barium esophagram because of significant dysphagia and respiratory distress since birth. The radiograph was highly suggestive of a double aortic arch. Surgical repair was performed through a left thoracotomy with division of a left atretic aortic arch. The patient had persistent stridor for 8 months postoperatively and then was referred for a pediatric otolaryngology evaluation. Microlaryngoscopy and bronchoscopy was performed at this time, revealing significant posterior and left lateral distal tracheal compression that was pulsatile in nature (Fig 3). An MRI scan was obtained and demonstrated a retroesophageal descending aorta with significant posterior tracheal compression (Fig 4). Tracheotomy was required due to increased stridor and oxygen desaturation. Microlaryngoscopy and bronchoscopy performed 4 months postoperatively revealed minimal improvement.

Case report no. 3. A 6-month-old infant with Down syndrome and atrial septal defect underwent an evaluation for failure to thrive. The patient was noted to have positional stridor, which was worse while lying on his back. Flexible laryngoscopy revealed significant laryngomalacia with left true vocal cord paralysis. Microlaryngoscopy and bronchoscopy demonstrated significant prolapse of the left arytenoid into the laryngeal lumen on inspiration. Distal tracheal compression was also noted (Fig 5). An MRI scan was obtained revealing no evidence of a vascular ring. A chest radiugraph, however, revealed an enlarged heart with increased pulmonary vasculature. A repeat MRI scan was obtained 8 months later, demonstrating significant distal tracheobronchial compression due to dilated pulmonary arteries (Fig 6). The patient underwent a left laser arytenoidectomy, and 1 week postoperatively underwent repair of his atrial septal defect through a thoracotomy approach. Eight months postoperatively the patient is doing well with no respiratory difficulty.

\section{DISCUSSION}

Numerous vascular anomalies involving the great vessels of the mediastinum can lead to tracheobronchial vascular compression. Their embryologic development is complex and small aberrations during embryogenesis may lead to significant vascular anomalies. A precisely timed sequence of selected migration and obliteration of portions of the first through sixth pairs of branchial arches leads to the development of the completed great vessels and their branches by week 8 of embryogenesis. The fourth and sixth branchial arches are the primary contributors to the vessels of the aortic arch. Detailed descriptions of this embryologic process have been published previously. ${ }^{1,3,10}$

Vascular anomalies of the great vessels may occur either as complete rings encircling the trachea and esophayus or as slings (incomplete rings),

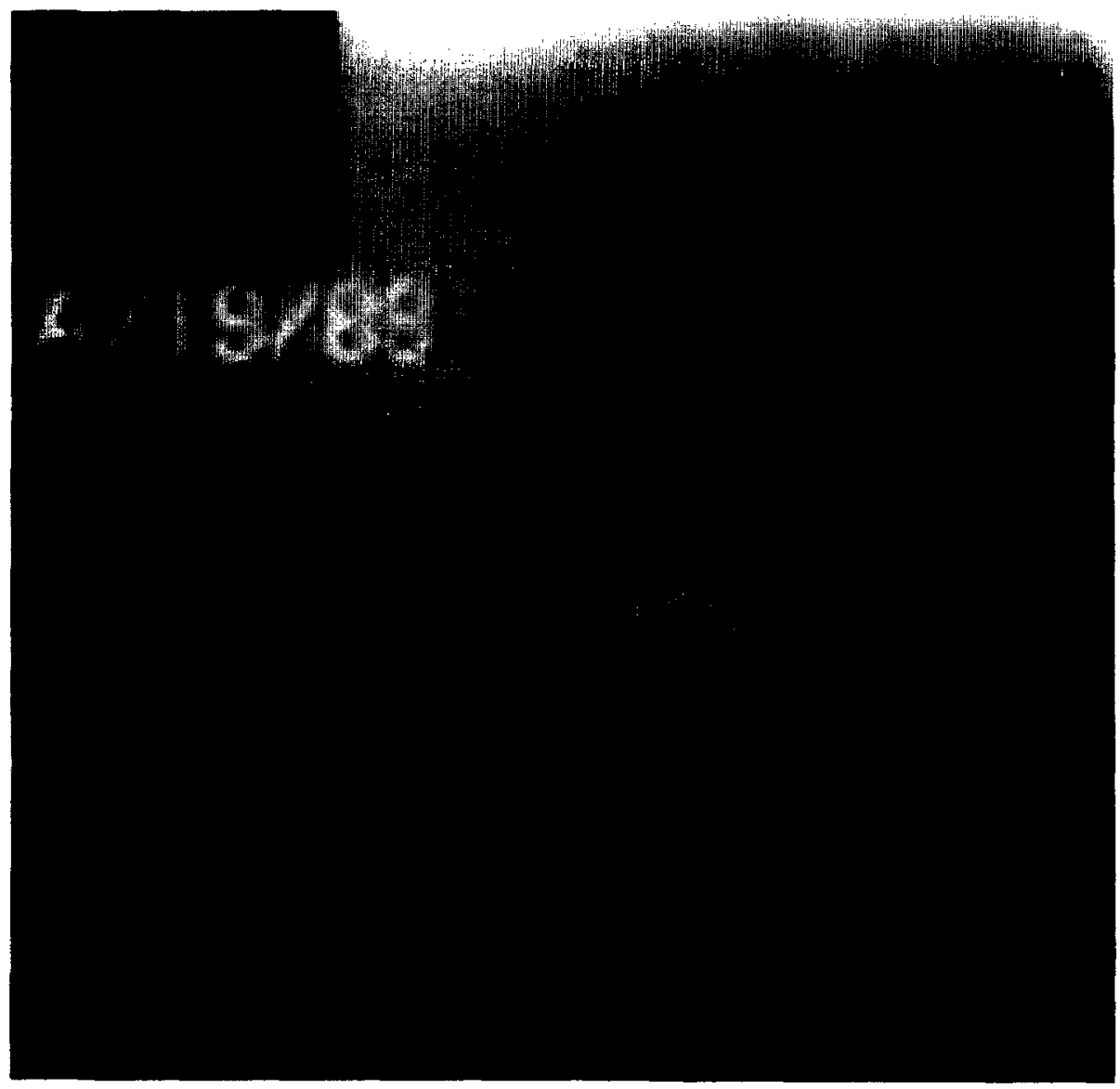

Figure 3. Endoscopic appearance of distal tracheal compression in case no. 2 


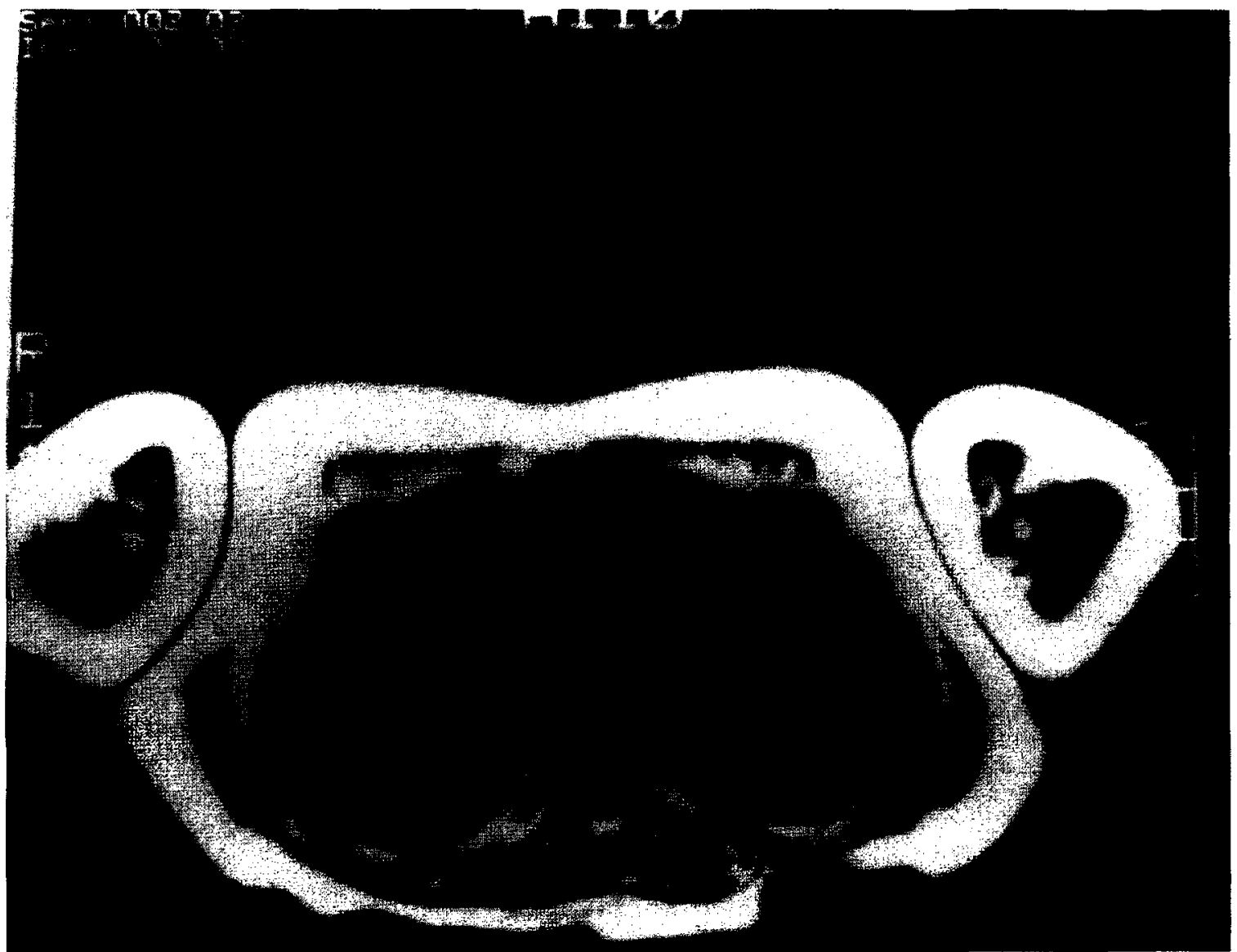

Figure 4. Magnetic resonance imaging scan of case no. 2 demonstrating a double aortic arch with a retroesophageal descending aorta.

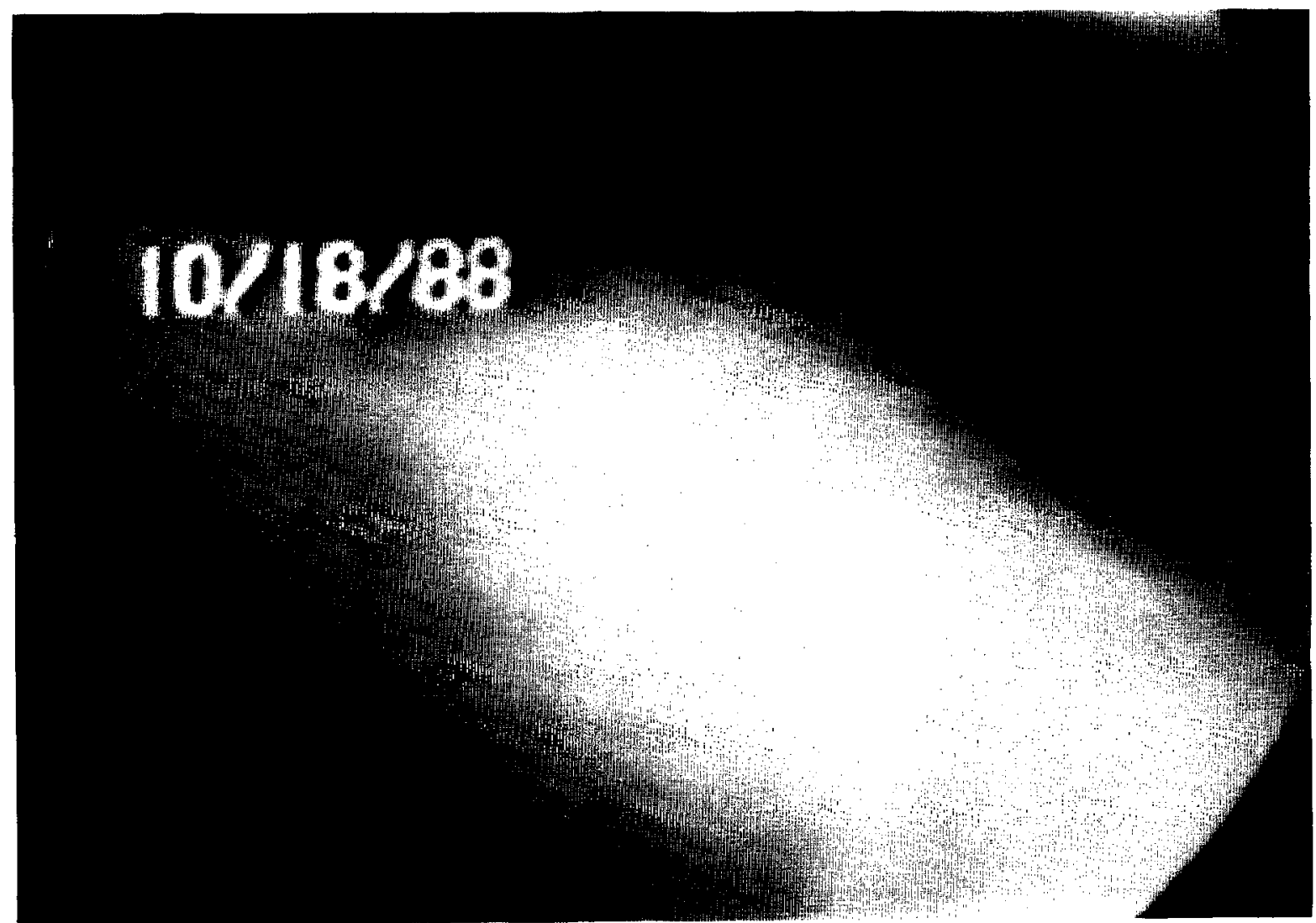

Figure 5. Endoscopic appearance of distal tracheal compression in case no. 3 caused by dilated pulmonary arteries. 


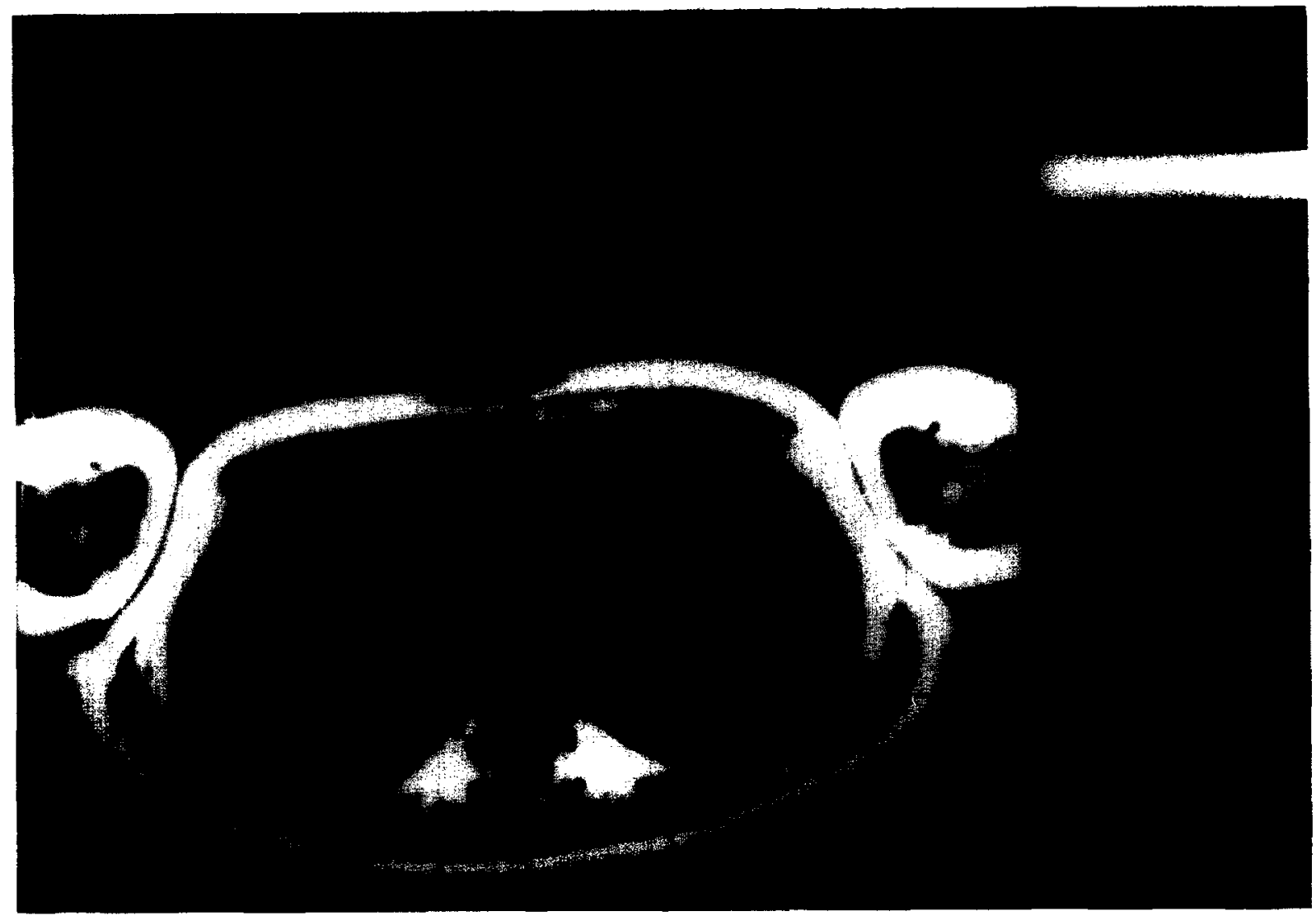

Figure 6. Magnetic resonance imaging scan reveals severe distal tracheobronchial compression due to dilated pulmonary arteries.

which usually cause less severe airway compression. Table 1 lists the most commonly encountered vascular rings and slings.

\section{Vascular Rings}

Double aortic arch. This is the most common vascular anomaly to cause severe airway compression in the nconate necessitating surgical intervention. This entity was first described by von Siebold in $1837^{3.11}$ and results from persistence of the right ventral fourth branchial arch forming a second, larger aortic arch. In $80 \%$ of cases of double aortic arch, the left arch is smaller and may even be atretic or nonpatent. ${ }^{1}$ The two arches join into a common aorta, which usually descends in the normal left-sided position (Fig 7). As was noted in our first case presentation, the descending aorta may lie in a retroesophageal position causing significant airway compression from the posterior region that may not be relieved with division of the double arch. Feeding difficulties may also result from this anomaly and a gastrostomy may be necessary.

Patients with a double aortic arch usually present in the early neonatal period. Airway obstruction may be severe, manifesting as stridor with episodes of cyanosis and, occasionally, apnea. Feeding difficulties may also be evident due to significant esophageal compression.
Bronchoscopic examination reveals bilateral pulsatile tracheal wall compression. A significant posterior component is evident in cases of retroesophageal aorta. Surgical intervention consists of a thoracotomy and division of the nondominant arch, usually the left. The ligamentum or ductus arteriosum is commonly interrupted at this time. Persistent tracheomalacia may be a postoperative problem and may require tracheotomy. Due to persistent tracheomalacia and persistent retroesophageal aorta, tracheotomy was necessary to relieve airway obstruction in one of the patients presented.

Right aortic arch. A persistent right aortic arch represents a fourth branchial vessel anomaly, which is usually obliterated during embryogenesis. If present, there is a high likelihood of associated congenital heart disease or a vascular ring anomaly. ${ }^{12}$ A right-sided arch with a descending right aorta in itself does not contribute to airway compromise. However, if the right arch passes retroesophageal to become a descending left aorta associated with a persistent ligamentum arteriosum, a constricting ring results, causing significant airway compromise (Fig 8). A right aortic arch with a right descending aorta associated with an aberrant left subclavian artery and a persistent ligamentum arteriosum may also lead to a constricting ring (Fig 9). Both these situations gener- 
TABLE 1. Vascular Anomalies Causing Tracheobronchial Compression

Vascular rings

Double aortic arch

Right aortic arch associated with

Ligamentum arteriosum

Retroesophageal or right descending aorta

Aberrant left subclavian artery Vascular slings

Anomalous innominate artery

Pulmonary artery sling

Aberrant right subclavian artery

ally result in less airway compromise than a true double aortic arch; however, surgical intervention is required in many cases. Clinical symptoms usually appear later in cases of a double aortic arch. A chest radiograph will often reveal a right aortic arch, and bronchoscopy will demonstrate pulsatile right tracheal wall compression. Surgical intervention usually consists of division of the ligamentum arteriosum or ductus arteriosum.

\section{Vascular Slings}

Innominate artery compression of the trachea. Gross and Neuhauser ${ }^{13}$ first reported this entity in 1948. Since that time numerous reports concerning innominate artery compression of the trachea have appeared..$^{7,8,14-16}$ In the normal anatomic situation, the innominate artery passes anterior to the trachea from left to right, usually originating completely or partially to the left of the trachea.
This was confirmed radiographically by Strife et al in $1981 .{ }^{14}$ Patients with innominate artery compression usually present in infancy, although older children may be affected. A case of a 15year-old with innominate artery compression manifesting as exercise intolerance was reported by Myer et al in 1989. ${ }^{8}$ The most common presenting symptoms are expiratory stridor and recurrent cough. Apnea is occasionally described, as well as recurrent bronchopulmonary infections. Increased respiratory efforts during periods of ugitation or stress may precipitate periods of reflex apnea or respiratory arrest.

Several theories have been postulated regarding the pathogenesis of innominate artery compression, including thymic pressure, crowding of the anterior mediastinum, and the presence of a short, taut innominate artery. ${ }^{8}$ Most patients with innominate artery compression do not require surgery. The absolute criteria for surgical intervention include reflex apnea, failure of medical management after 48 hours (intubation, steroids, antimicrobials), and repeated episodes of tracheobronchitis or bronchopneumonia, or both. Patients with concomitant airway anomalies such as repaired tracheoesophageal fistula with tracheomalacia, concomitant subglottic stenosis, asthma, cystic fibrosis, recurrent cough, tracheomalacia or laryngomalacia, and exercise intolerance are relative indications for surgery.

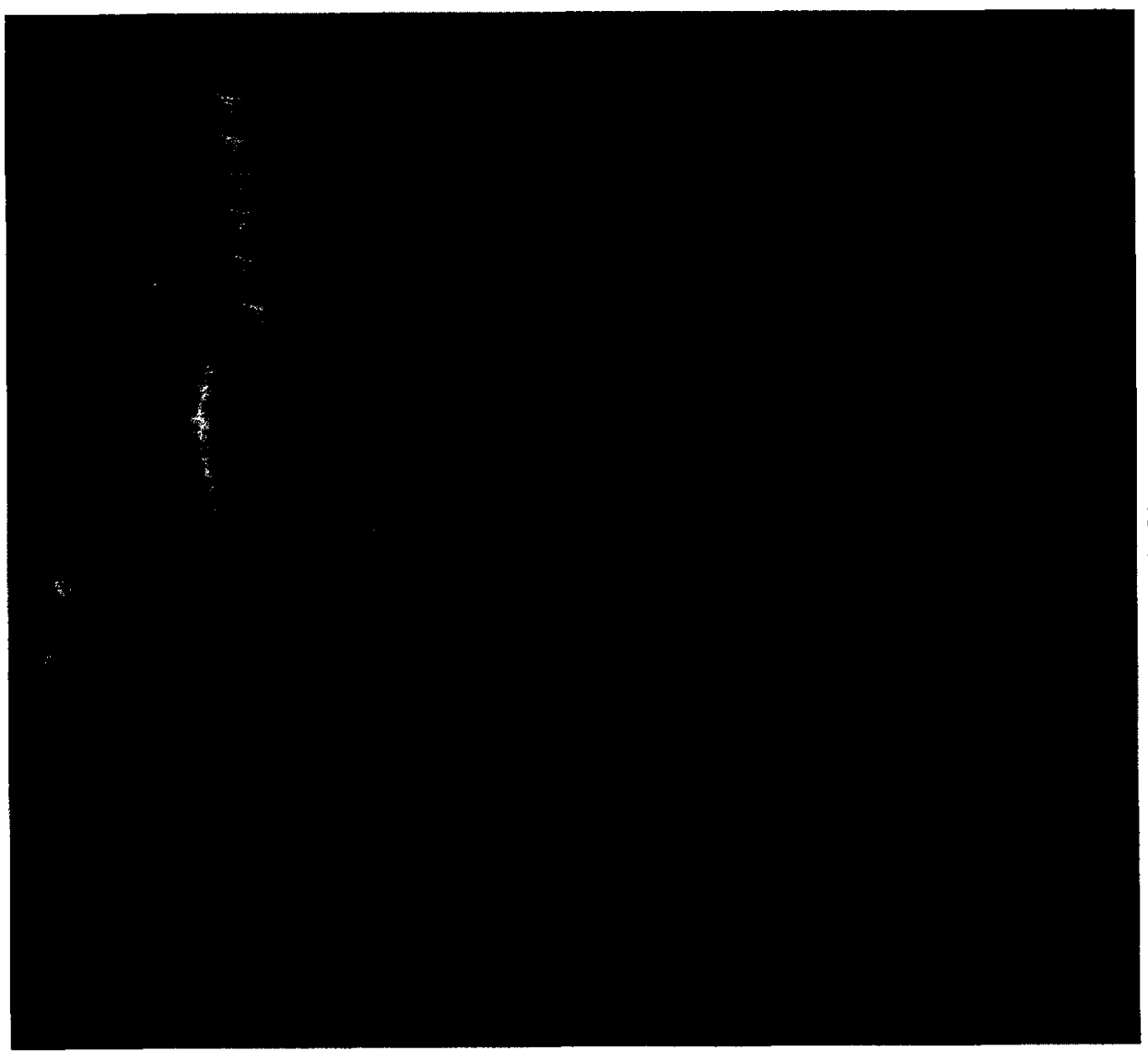

Figure 7. Double aortic arch The inset demonstrates areas of tracheosophageal compression. 


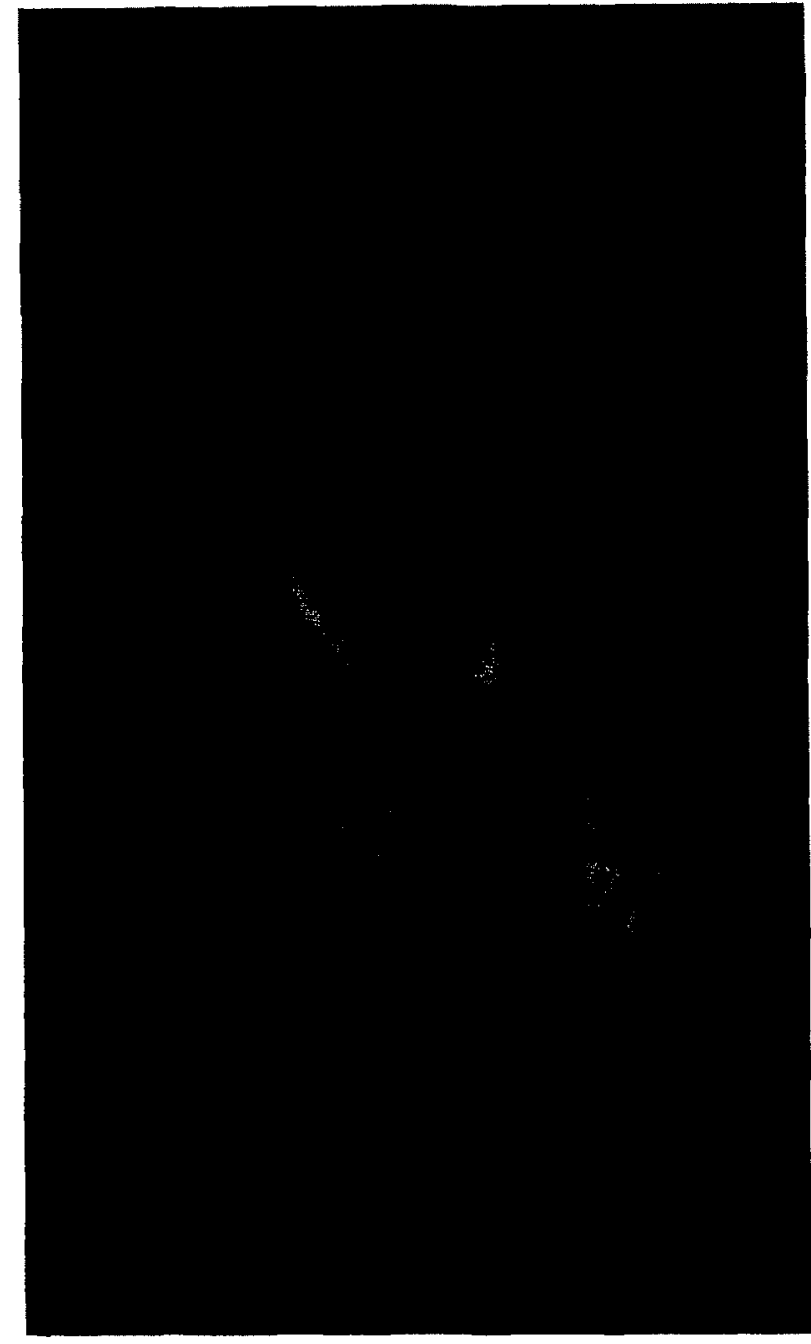

Figure 8. Right aortic arch anomaly associated with retroesophageal aorta descending on the left side with a ligamentum arteriosum forming a constricting ring.

A barium esophagram is of minimal diagnostic value because the innominate artery compresses the trachea anteriorly and there is no esophageal indentation. We have found microlaryngoscopy, bronchoscopy, and MRI scanning to be the diagnostic modalities of choice with this vascular anomaly. The surgical managment of this condition has evolved over the past 20 years. The standard therapy has been suspension of the innominate artery from the sternum; however, at our institution, reimplantation of the innominate artery on the proximal aorta has proven highly successful (Fig 10).

Pulmonary vascular sling. This vascular sling, although very uncommon, is the most symptomatic of the noncircumferential vascular anomalies. This entity results from the failure of development of the left sixth brachial arch, with the left pulmonary artery arising as a collateral of the right pulmonary artery coursing between the trachea and esophagus proximal to the right main-

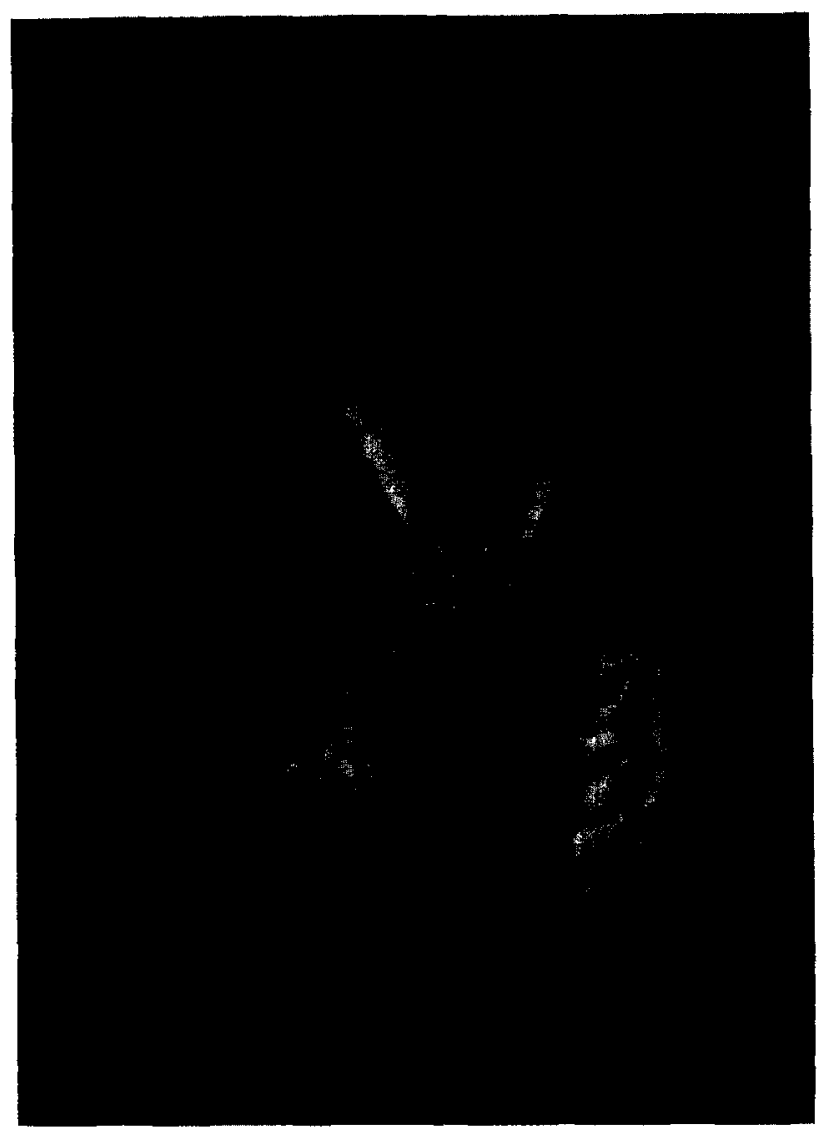

Figure 9. Aortic arch arising and descending on the right side with anomalous left subclavian artery and ligamentum arteriosum forming a constricting ring.

stem bronchus (Fig 11). This commonly results in significant compromise of the right mainstem bronchus. These patients usually have atelectasis and obstructive emphysema on the right side. Any infant developing obstructive emphysema of unknown etiology should undergo evaluation for this vascular anomaly. Stridor and significant respiratory distress are the common presenting symptoms. In addition, patients may have recurrent pulmonary infections and hyperinflation of the right lung. The diagnosis is made by endoscopy that reveals a nonpulsatile compression of the proximal right mainstem bronchus. Confirmation may be provided by MRI scanning. Fifty percent of patients with a pulmonary vascular sling have associated distal tracheal stenosis with complete tracheal rings. ${ }^{1}$

Aberrant right subclavian artery. This is the most common vascular anomaly overall, occurring in approximately 1 of 200 people. ${ }^{17}$ Though very few of these are actually symptomatic, affected individuals may present with dysphagia. In this vascular anomaly, the right subclavian artery arises independently from the distal part of the descending aortic arch rather than from the innominate artery. The artery then courses behind 


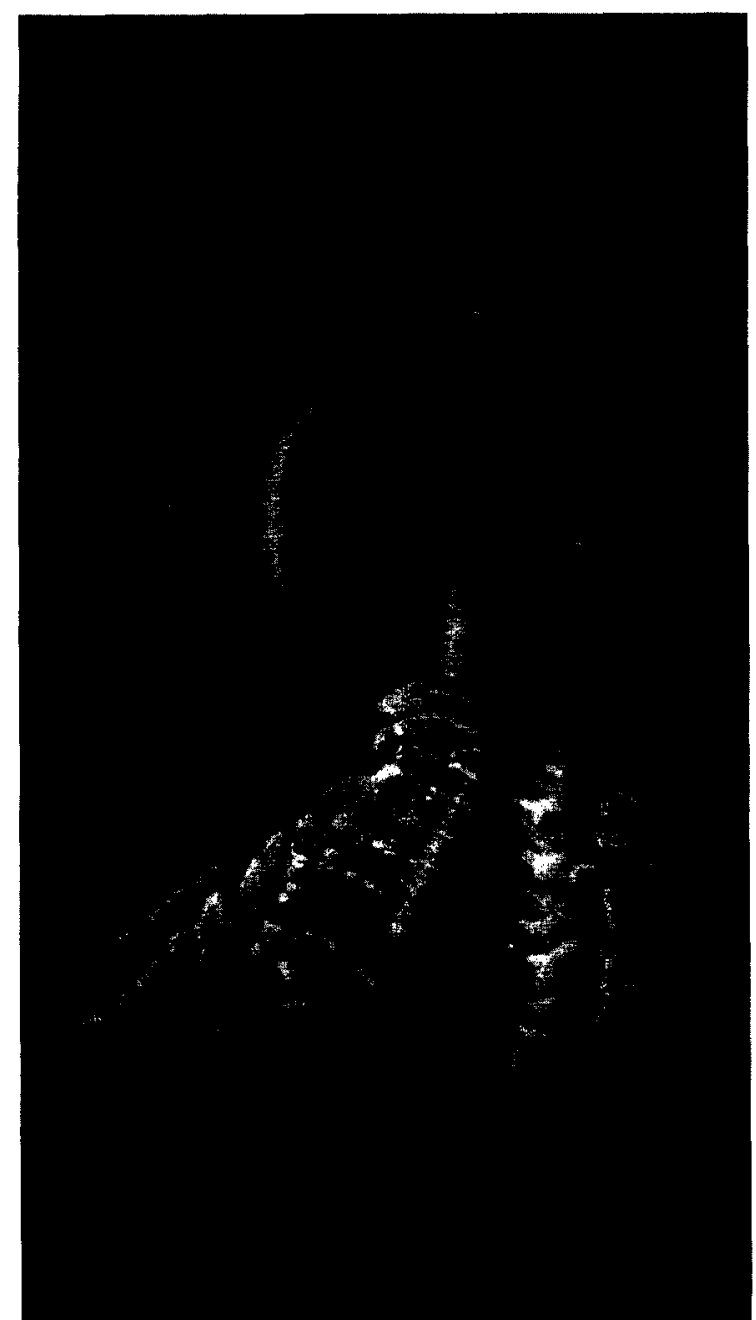

Figure 10. Innominate artery compression before and after surgical reimplantation on the proximal aortic arch.

the esophagus and upward, compressing the posterior esophageal wall. Very rarely does this result in airway compromise. The diagnosis is usually made by barium swallow and confirmed by esophagoscopy where a pulsatile posterior esophageal mass is noted. In severe cases, ligation of the artery may be necessary.

Tracheobronchial compression due to dilated pulmonary arteries in children with congenital heart disease has been described. ${ }^{18-20}$ In most cases, respiratory distress in children with heart disease is due to congestive heart failure, pneumonia, or compression of the lung parenchyma. Dilated and hypertensive pulmonary arteries may impinge on the tracheobronchial tree, usually in three specific sites: (1) the region where the left pulmonary artery arches over the left mainstem bronchus, (2) the area where the right pulmonary artery passes over the junction of the right bronchus intermedius and middle lobe bronchus, and (3) the left lateral aspect of the trachea immediately proximal to the carina where the aorta nor- mally places a slight indentation in the tracheal wall. Dilated hypertensive pulmonary arteries can further displace the aorta medially, accentuating the compression at this site. Compression of the left mainstem bronchus is further intensified in the presence of left atrial enlargement. The left atrium can impinge on the carina itself in cases of severe atrial enlargement.

The pulmonary arterial system is usually dilated in the presence of pulmonary arterial hypertension, although this is not always the case. Pulmonary artery hypertension may occur in simple acyanotic congenital heart defects such as ventricular septal defect or patent ductus arteriosus. Pulmonary arterial dilatation in the absence of hypertension may be present in cases of atrial septal defect, pulmonary valvar stenosis, and Tetralogy of Fallot with absent pulmonary valve ${ }^{18}$ Bronchial obstruction is rare in patients with simple atrial septal defect; however, this was the situation in our last case presentation. Table 2 lists congenital cardiac defects that may result in pulmonary arterial dilatation and subsequent tracheobronchial compression. Corrective surgery for a cardiac anomaly such as atrial septal defect may result in relief of the compression as occurred with the case presented. The importance of correcting associated airway anomalies was demonstrated by this patient's improvement after laser arytenoidectomy performed prior to cardiac surgery.

The diagnostic evaluation of vascular compression of the trachea has been evolving over recent years. Magnetic resonance imaging scanning has become the diagnostic modality of choice in many institutions for evaluating these lesions, ${ }^{7,9}$ Whereas a barium esophagram may be diagnostic for certain vascular rings, MRI better demonstrates the regional anatomy, which is extremely important if surgical intervention is anticipated. Some of the vascular rings and slings that are not as constricting as a double aortic arch may not be demonstrated on a barium esophagram.

Chest radiography remains extremely important in the evaluation of these patients because the cardiac silhouette and pulmonary vasculature can demonstrate evidence of cardiac disease that in itself may be leading to compression of the tracheobronchial tree. In patients with lobar emphysema, persistent or recurrent pneumonia, or gen-

TABLE 2. Pulmonary Artery Dilation

With pulmonary artery hypertension Ventricular septal defect

Patent ductus arteriosus

Without pulmonary artery hypertension Atrial septal defect Tetralogy of Fallot 


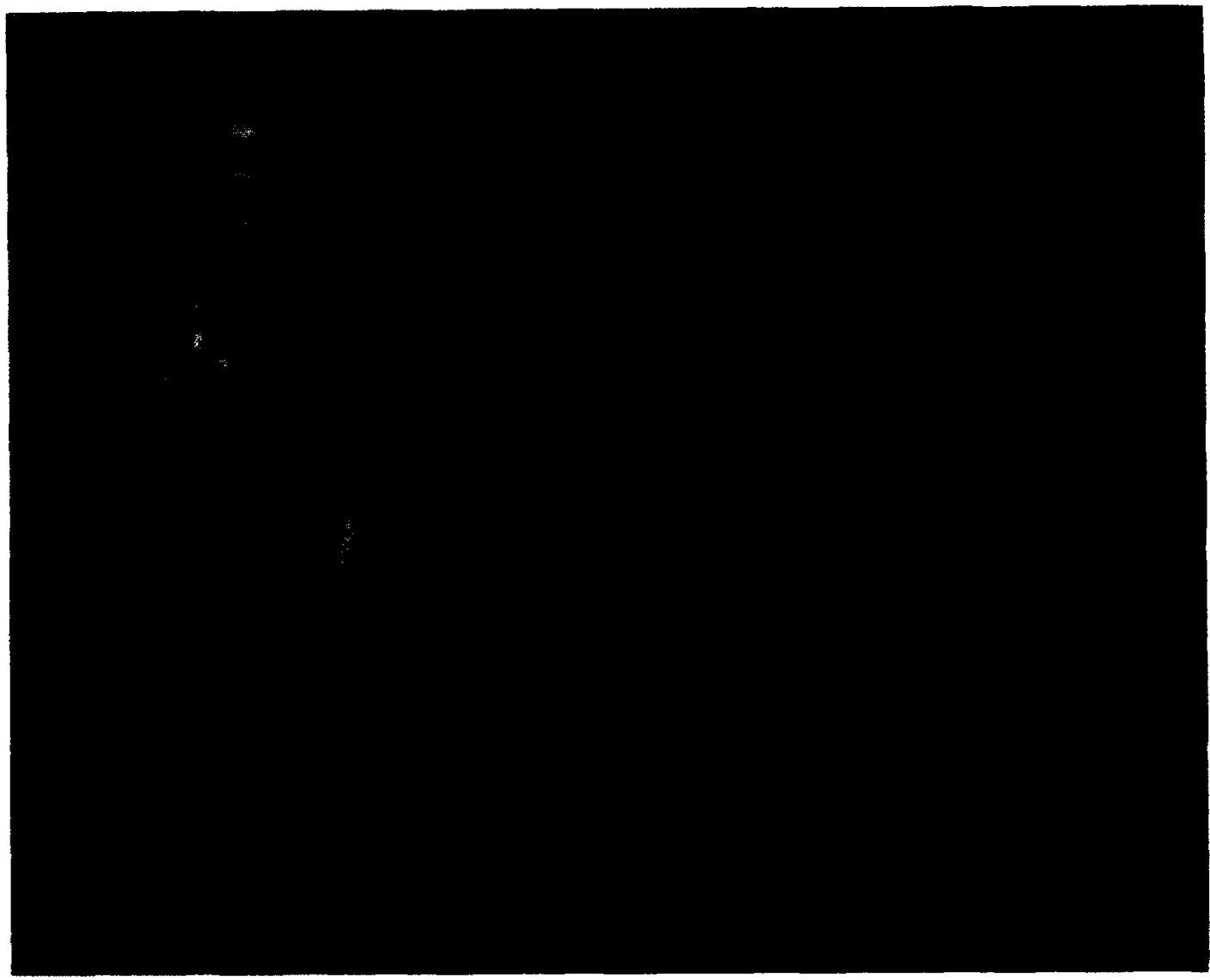

Figure 11. Pulmonary artery sling arising as a collateral of the right pulmonary artery coursing between the trachea and esophagus proximal to the right mainstem bronchus. The upper inset demonstrates the region of tracheoesophageal compression. The lower inset reveals an associated tracheal stenusis with complete tracheal rings.

eralized hyperinflation of one or both lungs, vascular tracheobronchial compression should be seriously considered in the diagnosis.

Flexible laryngoscopy is an important component of the diagnostic evaluation. Dynamic abnormalities such as vocal cord mobility and laryngomalacia may be assessed in addition to static conditions such as subglottic stenosis or glottic and supraglottic masses. All patients with suspected tracheobronchial compression should undergo rigid microlaryngoscopy and bronchoscopy to evaluate the location and extent of the compression and to evaluate associated airway anomalies. In one of our case presentations, there was an associated vocal cord paralysis with significant prolapse of an arytenoid cartilage on inspiration.

In addition to vascular compression, numerous other lesions may arise within the mediastinum and should be considered in the differential diagnosis of tracheobronchial compression (Table 3). Bony thorax abnormalities such as pectus excavatum may also cause significant tracheal compression. ${ }^{21}$ Magnetic resonance imaging scanning is useful in differentiating vascular compression from other etiologies of tracheobronchial narrow- ing. Figure 12 is the MRI scan of an 8-year-old patient who presented with airway obstruction and was found to have mediastinal lymphoma.

\section{CONCLUSION}

Three unusual cases of tracheobronchial vascular compression have been presented. Although surgical intervention may be corrective in most cases of vascular compression, persistent tracheomalacia or persistent vascular compression may necessitate tracheotomy for a prolonged period. The importance of cardiac evaluation in patients with suspected tracheobronchial compression is stressed because corrective cardiac surgery may

TABLE 3. Nonvascular Causes of Tracheobronchial Compression

\begin{tabular}{ll}
\hline \multicolumn{1}{c}{ BENIGN } & MaLignant \\
\hline $\begin{array}{l}\text { Bronchogenic cyst } \\
\text { Lymphangioloma } \\
\text { Hemangioma }\end{array}$ & Lymphoma \\
Teratoma & Carcinoid tumor \\
Hamartoma & \\
Lipoma & \\
\hline
\end{tabular}




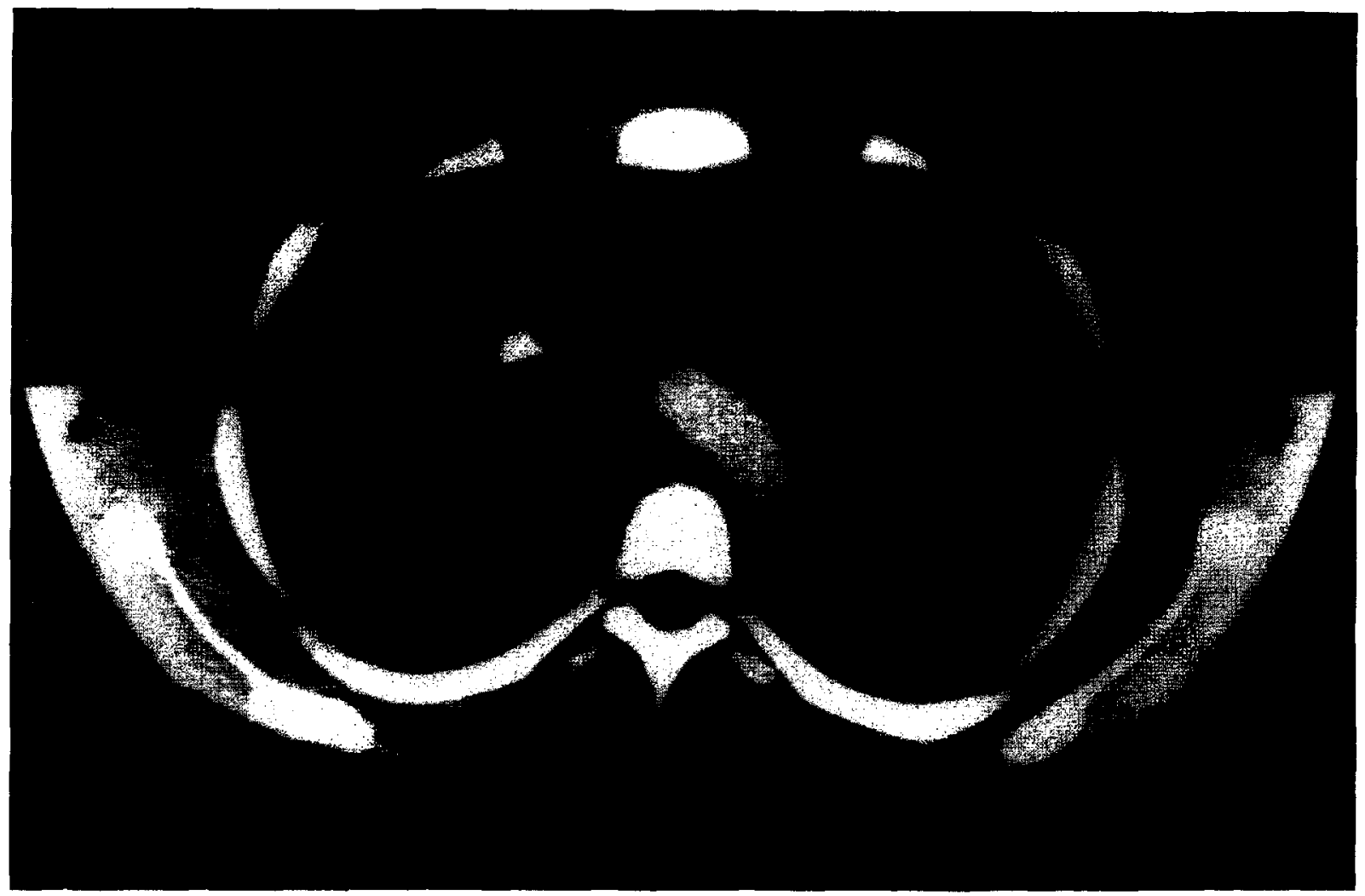

Figure 12. Magnetic resonance imaging image of mediastinal lymphoma with significant tracheal compression.

result in relief of the compression. The otolaryngologist should be aware of these entities and be prepared to initiate a diagnostic evaluation because the majority of these patients will present with airway compromise. Close communication between the otolaryngologist, pediatrician, radiologist, and anesthesiologist is extremely important in the management of these complicated problems.

\section{References}

1. Smith RJH, Smith MCF, Glossop LP, et al: Congenital vascular anomalies causing tracheoesophageal compression. Arch Otolaryng 1984; 110:82-87

2. Kraus DH, Hayes jD, Tucker HM: Vascular ring anomaly. Head Neck Surg 1989; 11:170-173

3. Keith $\mathrm{HH}$ : Vascular rings and tracheobronchial compression in infants. Pediatr Ann 1977; 6:540-549.

4. Vallette RC, Arensman RM, Falterman KW, et al: Tracheoesophageal compression syndromes related to vascular ring. South Med J 1989; 82:338-341

5. Backer CL. Ilbawi MN, Idriss FS, et al: Vascular anomalies causing tracheoesophageal compression. Review of experience in children. J Thoracic Cardiovasc Surg 1989; 97:725731

6. Fearon B, Shortrud R: Tracheobronchial compression by congenital cardiovascular anomalies in children. Ann Otol Rhinol Laryngol 1963; 72:949-969

7. Myer CM III, Auringer ST, Wiatrak BJ, et al: Magnetic resonance imaging of the diagnosis of innominate artery compression of the trachea. Arch Otolaryng Head Neck Surg 1990; 116:314-316

8. Myer CM III, Wiatrak BJ, Cotton RT, et al: Innominate artery compression of the trachea: Current concepts. Laryngoscope $1989 ; 99 ; 1030-1034$
9. Kersting-Sommerhoff BA, Sechtem UP, Fischer MR, et al: MR imaging of congenital anomalies of the aortic arch. Am ) Radiol 1987; 149:9-13

10. Harley HRS: The development and anomalies of the aortic arch and its branches. Brit J Surg 1959; 46:561-573

11. Lincoln JCR, Deverall PB, Stark J, et al: Vascular anom. alies compressing the oesophagus and trachea. Thorax 1969; 24:295-306

12. Steweart JR, Kincaid OW, Titus JL: Right aortic arch: Plain film diagnosis and significance. Am J Roentgen 1966; 97:377-389

13. Gross RE, Neuhauser EBD: Compression of the trachea by anolous innominate artery: An operation for its relief. Am J Dis Child 1948; 75:570-574

14. Strife JL, Baumel AS, Dunbar JS: Tracheal compression by the innominate artery in infancy and childhood. Radiology $1981 ; 139: 73$

15. Mustard WT, Bayliss CE, Fearon B: Tracheal compression by the innominate artery in children. Ann thorac Surg $1969 ; 8: 312$

16. Welz A, Reichert B, Weinhold C, et al: Innominate artery compression of the trachea in infancy and childhood: Surgical therapy justified? Thorac Cardiovas Surg 1984; $32: 85$

17. Gooding CA: Angiography of the thoracic aorta and its branches. in Gyepes MT (ed) Angiography in Infants and Children. New York NY. Grune \& Stratton, 1974, p 58

18. Berlinger NT, Lucas RV, Foker J: Pulmonary arteriopexy to relieve tracheobronchial compression by dilated pulmonary arteries. Ann Otolaryng Rhinol Laryngol 1984; 93:473-476

19. Dailey ME, O'Laughlin MP, Smith RJH: Airway compression secondary to left atrial enlargement and increased pulmonary artery pressure. Int I Pediatric Otorhinolaryng $1990 ; 19: 33-44$

20. Berlinger NT, Long C, Foker J, et al: Tracheobronchial compression in acyanotic congenital heart disease. Ann Otolaryng Rhinol Laryngol 1983; 92:387-390

21. Andrews TM, Myer CM III, Gray SP: Abnormalities of the bony thorax causing tracheobronchial compression. Int J Pediatric Otorhinolaryng 1990; 19:139-144 